\title{
EVALUACIÓN DE PROYECTOS EN INFLACIÓN ${ }^{*}$
}

Ignacio Vélez Pareja **

\section{RESUMEN}

Este artículo ilustra, por medio de varios ejemplos, que el valor presente net, VPN, que se utiliza para evaluar proyectos de inversión se debe calcular basado en precios corrientes. Ha existido la práctica muy utilizada de evaluar proyectos a precios constantes con una gran cantidad de "hoy" innecesarias sobre simplificaciones. Se presenta un ejemplo donde se muestra que la metodología de precios constantes (cero aumento de precios del año $0 \mathrm{y}$ tasa de descuento real) sesga la evaluación hacia sobrevaluar los proyectos y existe el riesgo potencial de aceptar proyectos malos como buenos.

Palabras clave: Evaluación de proyectos, ingeniería económica, valor presente neto, VPN, inflación, metodología de precios constantes, metodología de precios corrientes, metodología de precios nominales, flujo de caja libre, estados financieros proforma, metodología de precios relativos.

\section{AbSTRACT}

This article uses different examples to show why Net Present Value used project for assessment should be calculated current prices. It has been a widespread practice to assess projects at constant prices by making use of unnecessary oversimplifications. An example shows that the constant price methodology (zero increase in prices of year 0 and real discount rate) makes assessment up ward biased makes is possible to take a go decision on a bad project mistaken a good one.

Key word: Project evaluation, engineering economy, NPV, Net Present Value, inflation, constant price methodology, current price methodology, nominal price methodology, free cash flows, pro-forma financial statements, relative prices.

\footnotetext{
* $\quad$ Este artículo fue publicado como Working Paper en Social Science Research Network bajo el título Project Evaluation in an Inflationary Environment y como capítulo 7 del libro Decisiones de inversión. Enfocado a la valoración de empresas, Ceja, 2001.

** Ingeniero industrial de la Universidad de los Andes, M. Sc. en ingeniería industrial de la Universidad de Missouri Columbia, Missouri (Estados Unidos), decano de Ingeniería Industrial del Politécnico Grancolombiano, Bogotá (Colombia).

E-mail: ivelez@poligran.edu.co
} 


\section{Introducción}

Hay abundante literatura pero superficial sobre cómo involucrar la inflación en la evaluación de un proyecto. Muchos de esos autores son de países desarrollados con bajas o casi nulas tasas de inflación. En esta medida, se explica por qué no se le hace un tratamiento detallado a este problema. Sin embargo, como se verá más adelante, la distorsión de la inflación al evaluar un proyecto llega a ser importante aun en economías con inflaciones de un dígito. Por esta razón, en este artículo se estudia en detalle el efecto de la inflación en la evaluación de proyectos. Se verán tres metodologías de uso muy difundido: precios corrientes, precios constantes y precios relativos.

Hay tres enfoques para hacer la evaluación de proyectos en condiciones inflacionarias:

i) Precios nominales o corrientes (precios proyectados)

ii) Precios constantes (se conoce también como inflación neutral)

iii) Pesos (o cualquier otra moneda) constantes (inflación no neutral en los precios descontando el efecto de la inflación proyectada)

El enfoque de precios corrientes o nominales proyecta los precios de los insumos y productos y descuenta los flujos de caja futuros a las tasas de descuento futuras nominales o corrientes. El enfoque de precios constantes no proyecta aumentos de precios, supone que los precios se mantienen iguales y constantes al precio del instante cero a través de toda la vida del proyecto, $\mathrm{y}$ descuenta los flujos de caja futuros a la tasa de descuento real. Por último, el enfoque de los pesos constantes proyecta los aumentos relativos de precios y descuenta los flujos de caja futuros con la tasa de descuento real. ${ }^{1}$ Muchos analistas de proyectos dicen que cualquiera de los enfoques produce los mismos resultados; la única precaución en que insisten es, según ellos, que se debe ser consistente: no se deben mezclar flujos de caja nominales o corrientes con tasas de descuento reales y viceversa. Esta recomendación es una sutil manera de decir que si hay consistencia, entonces cualquiera de los enfoques producirá una respuesta correcta. En general esto no es cierto como se demostrará en esta parte del artículo.

Cuando se revisa la literatura, en particular los libros de texto de finanzas y de evaluación de proyectos se encuentra que muchos autores no son lo suficientemente claros acerca de cuál es el enfoque apropiado para utilizar al evaluar proyectos en inflación. El resultado es que en la práctica, y esto es crítico en las economías con altas tasas de inflación, los analistas terminan evaluando los proyectos de manera equivocada. Por ejemplo, Canada y White (1980) sugieren que es necesario ser cuidadosos y consistentes: los flujos de caja a precios corrientes se deben descontar con tasas de descuento corrientes y los flujos de caja a precios constantes con la tasa de descuento real; sin embargo, reconocen que los impuestos y la depreciación introducen algún sesgo en el análisis.

Para una descripción típica de la forma de trabajar cifras a precios constantes se recomienda la lectura del capítulo 14 de un texto clásico de las facultades de economía (Lora, 1987). 
Brealey, Myers y Marcus (1995) sostienen que se obtiene el mismo VPN ya sea con flujos de caja a precios corrientes y tasas de descuento corrientes o con flujos de caja a precios constantes y tasas de descuento real y previenen al lector respecto a no mezclar tasas reales y flujos de caja a precios constantes y viceversa.

Levy y Sarnat (1982) apuntan a que la respuesta correcta se obtiene con cualquiera de los métodos y que la única precaución que se debe tener es no mezclar tasas de interés (de descuento) y flujos de caja. Sin embargo, dedican un buen espacio a demostrar que cuando hay impuestos, la depreciación introduce un sesgo hacia arriba (mayor VPN) cuando se trabaja con precios constantes.

Weston y Copeland (1992) presentan un ejemplo muy detallado y muestran que cuando no hay inflación (o cuando hay inflación neutral) los resultados son los mismos. Cuando la inflación no es neutral, los resultados difieren; sin embargo, el lector termina con la idea de que la decisión resultante es la misma (el ejemplo muestra dos VPN positivos y no previene al lector sobre la posibilidad de que puede llegar a ser contradictoria).

Damodaran (1996) presenta un ejemplo que muestra la equivalencia de trabajar con cualquier método y advierte al lector de no mezclar tasas y flujos de caja.

Dixon y Hufschmidt (1986) reconocen que la inflación neutral no se puede suponer (cuando se trabaja con precios constantes) y proponen trabajar con el aumento relati- vo de los precios. Creen que los resultados son idénticos una vez que se incluyan los aumentos relativos de los precios.

Por el contrario muy pocos autores hacen la suficiente claridad para comprometerse con el enfoque correcto: los datos proyectados para calcular los flujos de caja se deben hacer a precios nominales o corrientes y esos flujos de caja futuros deben descontarse con base en la tasa de descuento corriente o nominal; por ejemplo, Copeland, Koller y Murrin (1995), Van Horne (1997) y Vélez (1983) mencionan que para decisiones sobre reemplazo de equipo no resulta equivalente trabajar con precios corrientes y con precios constantes.

Estas últimas posiciones son muy importantes. Y lo son porque muchos creen (por lo menos, según lo que se encuentra en las recomendaciones de algunas instituciones financieras tales como el BID) que el procedimiento correcto es el de los precios constantes. Esta entidad apoya la metodología de precios constantes. Cabe anotar que algunos economistas argumentan que esta metodología sí puede implicar aumento de precios relativos, pero obviamente esto es una contradicción. Esta propuesta se analiza más adelante. ${ }^{2}$

Es necesario dejar constancia de que algunos economistas amigos insisten en que la metodología a precios constantes no implica aumentos de precios iguales a cero; según ellos esta metodología de precios constantes debe llamarse precios a pesos constantes, lo cual permite considerar cambios de precios relativos. Se debe insistir en que precios constantes implica mantener iguales los precios y pesos constantes significa deflactar por medio de un deflactor, que podría ser el IPC, lo cual dejaría implícitos los aumentos de precios relativos. Así mismo, se ha generaliza- 
El principal argumento que se utiliza para defender esta metodología es que no se incurre en los posibles errores al tratar de pronosticar la inflación o el aumento de los precios y que además se obtienen los mismos resultados. Lo primero sería aceptable hace cincuenta años, cuando los instrumentos de cálculo y de análisis eran muy precarios; hoy, con la disponibilidad de los computadores, este argumento pierde su validez. Lo segundo es una falacia que surge de un esquema que proyecta los flujos a precios corrientes y después los deflacta con una tasa de inflación $\mathrm{y}$, a su vez, deflacta la tasa de interés con la misma tasa de inflación; esto es aritmética elemental, pues al dividir numerador y denominador por la misma cifra, los resultados al calcular el VPN con precios corrientes y precios constantes (en este caso son pesos constantes) son idénticos. Pero la realidad es otra. Como se verá más adelante los precios no aumentan lo mismo que la inflación. Otra razón que aducen las entidades financieras es que se debe evitar la apreciación subjetiva sobre precios que puede hacer cada solicitante de crédito, lo que se puede subsanar fácilmente, si se tiene en cuenta que los bancos centrales o las oficinas de estadística de los países mantienen información de todos los sectores y subsectores de la economía, de manera que su departamento de estudios económicos podrían hacer cálculos de precios o de los aumentos de éstos, para imponerlos en cada caso o para evaluar si el cálculo que hace el usuario es irreal o no. Inclusive se podría pensar en establecer una franja de precios o de aumentos por sectores y subsectores de la economía.

Por otro lado, quienes defienden esta metodología de análisis parece que descono- cen los supuestos implícitos que se hacen al suponer la inexistencia de los aumentos de precios y la coincidencia en las decisiones alcanzadas por el análisis a precios corrientes y a precios constantes.

En este artículo se abordan en primer término los problemas que surgen en la evaluación y las condiciones que se deben cumplir para que las diferentes metodologías arrojen idénticos resultados. En particular se estudian en detalle los supuestos que hay detrás de la metodología de precios constantes con el fin de que sea idéntica a la de precios corrientes. En la segunda parte se abordan las tres metodologías: precios constantes, corrientes o nominales, relativos y de pesos constantes. Se muestra con un ejemplo complejo los resultados de evaluar con esas metodologías y el resultado idéntico entre precios corrientes y constantes cuando se cumplen las condiciones estipuladas en la parte segunda. En la tercera se exponen algunas consideraciones adicionales para justificar el uso de la metodología de precios corrientes; $y$ en la cuarta se presentan las conclusiones.

\footnotetext{
do el uso de precios constantes con cero aumento de precios, esto es, que los precios del año 0 se mantienen a lo largo de la vida del proyecto. Esto es equivocado. En los párrafos siguientes se estudia el problema que surge entre las dos metodologías (tres si se acepta la posición arriba planteada). Debo aclarar también que la metodología de precios constantes implica que los precios iniciales no cambian; la metodología de pesos constantes es diferente y supone que los precios sí cambian y este cambio se refleja en los llamados precios relativos. Se debe hacer énfasis en el análisis y crítica de la metodología de precios constantes porque está muy difundida, tanto para la evaluación social de proyectos, como para la evaluación privada de los mismos.
} 


\section{Problemas y soluciones}

Se debe garantizar que con las dos metodologías de precios constantes y de precios corrientes se obtengan resultados idénticos en el valor presente neto; en caso de no ser iguales, aunque tengan el mismo signo, con sólo variar el monto de la inversión inicial, se cambiaría la decisión. Esa situación se puede observar en el siguiente ejemplo.

\section{Ejemplo 1}

\section{Cuadro 1}

\begin{tabular}{|c|c|c|}
\hline & Valor presente neto \$ & Decisión \\
\hline A precios constantes & 1.000 & Aceptar \\
\hline A precios corrientes & 2.000 & Aceptar \\
\hline
\end{tabular}

Si se aumentara la inversión en el instante cero en 1.500, entonces la situación sería la siguiente:

\section{Cuadro 2}

\begin{tabular}{|c|c|c|}
\hline & Valor presente neto \$ & Decisión \\
\hline A precios constantes & -500 & Rechazar \\
\hline A precios corrientes & 500 & Aceptar \\
\hline
\end{tabular}

Se puede constatar Aquí se ve que independiente de la metodología y sin cambiar ninguno de los supuestos de ellas, se cambia la decisión. Por tanto, evaluar proyectos con una u otra metodología no produce necesariamente los mismos resultados. Alguna de las dos debe estar errada. La metodología de precios corrientes pretende modelar la realidad tal y como se espera que ocurra en el futuro; la otra implica unos supuestos y mezcla de cifras en pesos de diferente poder adquisitivo que, como se verá a continuación, deberá ser desechada por equivocada.

Hay un ejemplo detallado en el archivo de Excel FLUJO.XLS en varias hojas, disponible en http://www.javeriana.edu.co/decisiones y a ese ejemplo se hará referencia en este aparte del artículo. Se puede copiar desde la red y se aconseja estudiar esta parte acompañada de esas hojas de cálculo.

\subsection{Supuestos implícitos}

Los supuestos implícitos que hay detrás del análisis a precios constantes para que la decisión siempre coincida, esto implica que los resultados del VPN a corrientes sea idéntica al resultado del VPN a constantes, con el análisis a precios corrientes son los siguientes:

A. ¡No hay impuestos!

B. La reinversión de los flujos de caja intermedios durante la vida del proyecto 
se debe hacer estrictamente a la tasa de descuento

C. Los aumentos de precios que ocurrirán en la realidad (a precios corrientes) serán iguales a la inflación, incluida en la tasa de descuento nominal o corriente

D. Las ventas y pagos por bienes y servicios se hacen de estricto contado

E. No existe valor de salvamento del proyecto

F. No existen relaciones de elasticidad precio-demanda

G. La tasa de descuento a precios corrientes o nominales, debe ser exactamente igual a

(1+tasa real/1 +tasa de inflación)-1

$\mathrm{y}$ a precios constantes debe ser igual a $\mathrm{i}_{\mathrm{r}}$, la tasa de interés real.

Cada uno de estos supuestos o condiciones se analizan a continuación.

\subsubsection{Supuesto A}

¡No hay impuestos!

Cuando los impuestos existen, la depreciación y los intereses generan ahorros que se atribuyen al proyecto. En el caso de la depreciación sería idéntico en valor absoluto a precios constantes y a precios corrientes. Sin embargo el valor relativo es mayor a precios constantes que a precios corrientes. Por tanto el proyecto quedaría sobrevaluado, porque se subestima la renta gravable y con ello los impuestos por pagar. Ahora bien, como los impuestos son inevitables, si se supone que sí los hay, entonces debe suponerse que no hay depreciación ni financiación. Algunos autores que han tomado conciencia de ello lo que proponen es deflactar la depreciación, lo que significa manipulación de cifras sin ningún sentido económico.

En sentido analítico se puede hacer la siguiente consideración: si se supone que todos los ingresos y gastos registrados en un $\mathrm{P}$ y $\mathrm{G}$ correspondieran a operaciones de contado y que no existiera financiación, entonces el FCL después de impuestos sería: $^{3}$

$(S-D) *(1-T)+D=(1-T) S+T D$

Donde:

$S=$ Saldo de efectivo

$D=$ Depreciación

$T=$ Tasa de impuestos

Si no existe inflación, el VPN (expresado como $\mathrm{VPN}_{\mathrm{NI}}$ a la tasa de descuento real $\mathrm{i}_{\mathrm{r}}$ sería

$$
V P N_{N I}=\sum_{t=1}^{n} \frac{(1-T) S_{t}}{\left(1+i_{r}\right)^{t}}+\sum_{t=1}^{n} \frac{T D_{t}}{\left(1+i_{r}\right)^{t}}-I
$$

Si hay inflación $i_{f}$ y todos los precios y costos aumentan en ese porcentaje, entonces el saldo $S_{t}$ se aumenta en $\left(1+i_{f}\right)^{t}$ y el factor de valor presente se reduce por $\left(1+i_{\mathrm{f}}\right)^{t}$, esto es que el VPN con inflación $\left(\mathrm{VPN}_{\mathrm{I}}\right)$ es,

Este análisis se basa en Levy y Sarnat (1982) y se emplea la misma notación. 


$$
\begin{aligned}
& V P N_{I}=\sum_{t=1}^{n} \frac{(1-T) S_{t}\left(1+i_{f}\right)^{t}}{\left(1+i_{r}\right)^{t}\left(1+i_{f}\right)^{t}}+\sum_{t=1}^{n} \frac{T D_{t}}{\left(1+i_{r}\right)^{t}\left(1+i_{f}\right)^{t}}-I \\
& =\sum_{t=1}^{n} \frac{(1-T) S_{t}}{\left(1+i_{r}\right)^{t}}+\sum_{t=1}^{n} \frac{T D_{t}}{\left(1+i_{r}\right)^{t}\left(1+i_{f}\right)^{t}}-I
\end{aligned}
$$

$\mathrm{O}$ sea que se va a presentar una diferencia entre el VPN a precios constantes (sin inflación) y el VPN a precios corrientes (con inflación). Esta diferencia es:

$$
\sum_{t=1}^{n} \frac{T D_{t}}{\left(1+i_{r}\right)^{t}}-\sum_{t=1}^{n} \frac{T D_{t}}{\left(1+i_{r}\right)^{t}\left(1+i_{f}\right)^{t}}
$$

$$
\frac{\frac{T D_{t}}{\left(1+i_{r}\right)^{t}}}{\frac{T D_{t}}{\left(1+i_{r}\right)^{t}\left(1+i_{f}\right)^{t}}}=\left(1+i_{f}\right)^{n}
$$

La relación entre el valor presente del ahorro por depreciación a precios constantes y a precios corrientes es $\left(1+\mathrm{i}_{\mathrm{f}}\right)^{\mathrm{n}}$. Esto es la magnitud del mayor valor en que se sesga el análisis de VPN cuando se trabaja en precios constantes y en cuanto a ahorro en impuestos por depreciación.

\section{Cuadro 3}

Efecto de la inflación sobre 1 peso en ahorro de impuesto por depreciación en diferentes períodos

\begin{tabular}{|c|c|c|c|c|c|}
\hline Años & $\mathbf{1}$ & $\mathbf{3}$ & $\mathbf{5}$ & $\mathbf{6}$ & $\mathbf{1 0}$ \\
\hline $0 \%$ & $0,0 \%$ & $0,0 \%$ & $0,0 \%$ & $0,0 \%$ & $0,0 \%$ \\
\hline $1 \%$ & $1,0 \%$ & $3,0 \%$ & $5,1 \%$ & $6,2 \%$ & $10,5 \%$ \\
\hline $2 \%$ & $2,0 \%$ & $6,1 \%$ & $10,4 \%$ & $12,6 \%$ & $21,9 \%$ \\
\hline $2,50 \%$ & $2,5 \%$ & $7,7 \%$ & $13,1 \%$ & $16,0 \%$ & $28,0 \%$ \\
\hline $5 \%$ & $5,0 \%$ & $15,8 \%$ & $27,6 \%$ & $34,0 \%$ & $62,9 \%$ \\
\hline $7,50 \%$ & $7,5 \%$ & $24,2 \%$ & $43,6 \%$ & $54,3 \%$ & $106,1 \%$ \\
\hline $10 \%$ & $10,0 \%$ & $33,1 \%$ & $61,1 \%$ & $77,2 \%$ & $159,4 \%$ \\
\hline $12,50 \%$ & $12,5 \%$ & $42,4 \%$ & $80,2 \%$ & $102,7 \%$ & $224,7 \%$ \\
\hline $15 \%$ & $15,0 \%$ & $52,1 \%$ & $101,1 \%$ & $131,3 \%$ & $304,6 \%$ \\
\hline $17,50 \%$ & $17,5 \%$ & $62,2 \%$ & $124,0 \%$ & $163,2 \%$ & $401,6 \%$ \\
\hline $20 \%$ & $20,0 \%$ & $72,8 \%$ & $148,8 \%$ & $198,6 \%$ & $519,2 \%$ \\
\hline $22,50 \%$ & $22,5 \%$ & $83,8 \%$ & $175,9 \%$ & $237,9 \%$ & $661,0 \%$ \\
\hline
\end{tabular}


En el Cuadro 3 se puede observar que aun en economías de inflación menor que 5\% los efectos de sesgo por la inflación, en lo que se refiere a ahorros en impuestos por depreciación, a favor de la metodología de precios constantes es mayor que $5 \%$ en períodos tan cercanos como tres años para una inflación de $2 \%$ y cinco años para una inflación de $1 \%$.

Si se analiza el valor presente de una cuotas uniforme de un peso durante cinco años y se compara ese valor presente a tasa de interés real contra el mismo valor presente a tasa de interés corriente, se encuentra:

En el Cuadro 4 se muestra el error a favor del análisis a precios constantes por un peso en ahorros en impuestos por depreciación durante cinco años para diferentes combinaciones de tasas de interés reales y tasas de inflación. El cálculo está hecho como:

Valor presente de ahorro en impuestos por depreciación a tasa real

Valor presente de ahorro en impuestos por depreciación a tasa corriente

\section{Cuadro 4}

Error en valor presente de ahorro en depreciación: precios constantes frente a precios corrientes

\begin{tabular}{|c|c|c|c|c|c|c|}
\hline & \multicolumn{7}{|c|}{ Tasa de interés real } \\
\hline Inflación & $\mathbf{1 \%}$ & $\mathbf{3 \%}$ & $\mathbf{5 \%}$ & $\mathbf{6 \%}$ & $\mathbf{1 0 \%}$ & $\mathbf{1 2 \%}$ \\
\hline $0,0 \%$ & $0,00 \%$ & $0,00 \%$ & $0,00 \%$ & $0,00 \%$ & $0,00 \%$ & $0,00 \%$ \\
\hline $1,0 \%$ & $3,00 \%$ & $2,96 \%$ & $2,92 \%$ & $2,90 \%$ & $2,83 \%$ & $2,79 \%$ \\
\hline $2,0 \%$ & $6,04 \%$ & $5,96 \%$ & $5,87 \%$ & $5,84 \%$ & $5,68 \%$ & $5,61 \%$ \\
\hline $2,5 \%$ & $7,57 \%$ & $7,47 \%$ & $7,36 \%$ & $7,31 \%$ & $7,12 \%$ & $7,03 \%$ \\
\hline $5,0 \%$ & $15,38 \%$ & $15,16 \%$ & $14,94 \%$ & $14,84 \%$ & $14,43 \%$ & $14,23 \%$ \\
\hline $7,5 \%$ & $23,41 \%$ & $23,06 \%$ & $22,72 \%$ & $22,55 \%$ & $21,91 \%$ & $21,60 \%$ \\
\hline $10,0 \%$ & $31,65 \%$ & $31,16 \%$ & $30,69 \%$ & $30,45 \%$ & $29,56 \%$ & $29,13 \%$ \\
\hline $12,5 \%$ & $40,10 \%$ & $39,46 \%$ & $38,84 \%$ & $38,54 \%$ & $37,36 \%$ & $36,80 \%$ \\
\hline $15,0 \%$ & $48,75 \%$ & $47,94 \%$ & $47,16 \%$ & $46,78 \%$ & $45,32 \%$ & $44,62 \%$ \\
\hline $17,5 \%$ & $57,58 \%$ & $56,60 \%$ & $55,66 \%$ & $55,19 \%$ & $53,41 \%$ & $52,56 \%$ \\
\hline $20,0 \%$ & $66,59 \%$ & $65,43 \%$ & $64,30 \%$ & $63,75 \%$ & $61,64 \%$ & $60,63 \%$ \\
\hline $22,5 \%$ & $75,78 \%$ & $74,41 \%$ & $73,10 \%$ & $72,46 \%$ & $69,99 \%$ & $68,82 \%$ \\
\hline
\end{tabular}

Otra vez se puede observar que aun en economías con bajas tasas de inflación los efectos del sesgo de evaluar un flujo de caja con la metodología de precios constantes, en lo que se refiere a ahorros en impuestos por depreciación, se producen errores considerables.

El efecto de la depreciación sobre el VPN a precios constantes comparado con precios 
corrientes se puede apreciar en el ejemplo indicado en el Cuadro 3.

\section{Ejemplo 2}

Si la tasa de interés real es de $6 \%$, la inflación es de $10 \%$ y el aumento relativo de precios y costos es $1 \%$, entonces la tasa de descuento es de $16,6 \%$. El aumento de precios y costos es de $11,1 \%$. Los impuestos son de $40 \%$. Un P y G simplificado es

\section{Cuadro 5}

\begin{tabular}{|c|c|c|c|c|c|}
\hline & \multicolumn{2}{|c|}{ Precios constantes } & \multicolumn{2}{c|}{ Precios corrientes } & Aumento de cada partida \\
\hline Ventas & 100 & $100,0 \%$ & 111,1 & $100,0 \%$ & $11,1 \%$ \\
\hline Gastos & 50 & $50,0 \%$ & 55,55 & $50,0 \%$ & $11,1 \%$ \\
\hline Depreciación & 10 & $10,0 \%$ & 10 & $9,0 \%$ & $0,0 \%$ \\
\hline Utilidad & 40 & $40,0 \%$ & 45,55 & $41,0 \%$ & $13,9 \%$ \\
\hline Impuestos & 16 & $16,0 \%$ & 18,22 & $16,4 \%$ & $13,9 \%$ \\
\hline Utilidad neta & 24 & $24,0 \%$ & 27,33 & $24,6 \%$ & $13,9 \%$ \\
\hline
\end{tabular}

Si se supone que todas las operaciones se hacen de contado y los impuestos se pagan el mismo período en que se causan, entonces el flujo de caja neto será:

\section{Cuadro 6}

\begin{tabular}{|c|c|c|}
\hline Flujo & Precios constantes & Precios corrientes \\
\hline Año 1 & 34 & 37,33 \\
\hline Valor presente & 32,08 & 32,02 \\
\hline
\end{tabular}

Para el caso de pesos constantes sucede algo similar:

\section{Cuadro 7}

\begin{tabular}{|c|c|c|c|c|c|}
\hline $\begin{array}{c}\text { Estado de pérdidas } \\
\text { y ganancias }\end{array}$ & \multicolumn{2}{|c|}{ Pesos constantes } & \multicolumn{2}{c|}{ Precios corrientes } & Aumento de cada partida \\
\hline Ventas & 101 & $100,0 \%$ & 111,1 & $100,0 \%$ & $11,1 \%$ \\
\hline Gastos & 50,5 & $50,0 \%$ & 55,55 & $50,0 \%$ & $11,1 \%$ \\
\hline Depreciación & 10 & $9,9 \%$ & 10 & $9,0 \%$ & $0,0 \%$ \\
\hline Utilidad & 40 & $40,1 \%$ & 45,55 & $41,0 \%$ & $13,9 \%$ \\
\hline Impuestos & 16 & $16,0 \%$ & 18,22 & $16,4 \%$ & $13,9 \%$ \\
\hline Utilidad neta & 24 & $24,1 \%$ & 27,33 & $24,6 \%$ & $13,9 \%$ \\
\hline
\end{tabular}

Si se supone que todas las operaciones se hacen de contado y los impuestos se pagan el mismo período en que se causan, entonces el flujo de caja neto será: 
Cuadro 8

\begin{tabular}{|c|c|c|}
\hline Flujo & Pesos constantes & Precios corrientes \\
\hline Año 1 & 34,3 & 37,33 \\
\hline Valor presente & $\$ 32,36$ & $\$ 32,02$ \\
\hline
\end{tabular}

Se puede observar cómo el peso relativo de la depreciación es mayor en la columna de precios constantes y pesos constantes, y eso se refleja en menores impuestos relativos; lo cual, a su vez, se manifiesta en el valor presente. Aquí se ve con claridad que en este caso un proyecto a precios constantes queda mejor evaluado (VPN mayor) que ese mismo proyecto evaluado a precios corrientes. Y esto es grave porque es posible que muchos proyectos hayan sido aceptados como buenos, siendo malos. Muchas veces no se sabe por qué ocurren fracasos estruendosos en proyectos aparentemente recomendables. Una posible explicación se relaciona con que el autor tiene alguna intuición, sin datos empíricos formales para apoyarla: ${ }^{4}$ que muchos proyectos - públicos y privados - fracasan porque han sido aceptados con un VPN positivo calculado con la metodología de precios constantes y que si se hubieran evaluado a precios corrientes es posible que hubieran sido rechazados. Esto se debe estudiar de manera formal.

La expresión (4) es muy elegante y simple, pero es válida en un contexto muy restringido, tal como se indicó arriba. Se puede tratar de hacer un ajuste a los resultados de la metodología a precios constantes, restando la cantidad definida en (4), pero el problema es que sólo se puede hacer si el resultado calculado a precios corrientes $\left(\mathrm{VPN}_{\mathrm{I}}\right)$ se conoce. De hecho, de antemano no se sabe si la firma obtendrá o no los ahorros en impuestos (a precios constantes y corrientes). Estos se conocen una vez que los estados financieros proforma a precios corrientes y constantes se han construido, y para conocerlos se debe pronosticar la inflación. Es necesario saber si la firma paga o no impuestos $\mathrm{Si}$ el proyecto (o firma) no paga impuestos, no existirá ahorro en impuestos. Y evitar el pronóstico de la inflación era uno de los propósitos de la metodología a precios constantes.

Parte de esta diferencia se puede compensar si la evaluación de proyectos a precios constantes se hace con impuestos calculados sin tener en cuenta los ajustes por inflación y la evaluación de proyectos a precios corrientes se hace con impuestos calculados teniendo en cuenta los ajustes por inflación. Sin embargo, esta corrección puede que no sea suficiente. El lector puede comprobarlo con las cifras del archivo FLUJO.XLS (www.poligran.edu.co/decisiones).

Además, si se están comparando proyectos con diferente intensidad de inversión en activos fijos, entonces las diferencias son aun mayores.

\footnotetext{
Alguna evidencia aislada y muy restringida acerca de esto: en 1986 enseñé este tema en programa de posgrado para una siderúrgica muy importante en Colombia y se examinó esta hipótesis con dos proyectos que fracasaron. Se reconstruyó el estudio de la evaluación -hecha a precios constantes- con los datos disponibles de la época y los resultados finales -a precios corrientes- indicaron que los proyectos debieron ser rechazados.
} 


\subsubsection{Supuesto $B$}

La reinversión de los flujos de caja intermedios durante la vida del proyecto se debe hacer estrictamente a la tasa de descuento

Esto es que a precios corrientes la reinversión se debe hacer a la tasa de descuento corriente y a precios constantes a la tasa de descuento deflactada o real. Ello no siempre es posible en la realidad y por lo general no lo es. Cuando la evaluación de un proyecto se hace en una hoja de cálculo (que es el caso general en estos días) los excedentes de efectivo del FT se invierten a las tasas de mercado ${ }^{5}$. Una forma de garantizar este supuesto es eliminar el rubro de inversión de excedentes de liquidez en el flujo de fondos y al calcular el VPN, que supone reinversión automática a la tasa de descuento quedaría considerada la reinversión a esa tasa (véase el ejemplo ya mencionado).

Por lo general, la tasa a la cual se pueden invertir los excedentes es diferente a la de descuento y en particular a $\left(1+\mathrm{i}_{\mathrm{f}}\right)\left(1+\mathrm{i}_{\mathrm{r}}\right)-1^{6}$. Sin embargo, en el caso del ejemplo detallado las tasas de reinversión de los excedentes fueron las siguientes:

\section{Cuadro 9}

\begin{tabular}{|c|c|c|c|c|}
\hline Años & $\mathbf{1}$ & $\mathbf{2}$ & $\mathbf{3}$ & $\mathbf{4}$ \\
\hline$\left(1+\mathrm{i}_{\mathrm{f}}\right)\left(1+\mathrm{i}_{\mathrm{r}}\right)-1 \%$ & 29,32 & 30,38 & 28,26 & 27,20 \\
\hline Tasa de reinversión \% & 32,70 & 34,02 & 31,40 & 30,10 \\
\hline
\end{tabular}

\subsubsection{Supuesto $C$}

Los aumentos de precios que ocurrirán en la realidad (a precios corrientes) serán iguales a la inflación incluida en la tasa de descuento nominal o corriente.

Esta situación se denomina una inflación neutral, en la cual todos los precios y costos aumentan en igual proporción. Esto, a simple vista, es irreal, ya que día a día se observa que el comportamiento de los precios no es igual a la inflación. De hecho, la inflación es un promedio ponderado de los aumentos de una gran variedad de productos y servicios. Hay autores que reconocen este hecho y proponen utilizar los aumentos de precios relativos. (Véase Dixon y
Hufschmidt, p. 42). Esta solución no es suficiente para resolver el problema, pues quedan pendientes todos los demás puntos aquí mencionados.

Cuando en el archivo INFLACION.XLS se toman los datos del flujo de caja y se evalúa con tasa sin inflación y con los precios relativos, descontando la inflación (esto no se hace restando del aumento de precios la

Esto significa que en la realidad, el supuesto implícito de reinversión de los fondos en cuanto a que al calcular el VPN lleva implícita la reinversión de los flujos de caja intermedios a la tasa de descuento no es cierta: lo que se reinvierte es el excedente de liquidez que aparece en el FT y no los excedentes que aparecen en el flujo de caja libre del proyecto (FCL)

6 Recuérdese lo planteado por Fisher sobre las componentes de la tasa de interés del mercado. 
inflación, como lo hacen muchos, sino descontándola del aumento de precio: $((1+$ aumento de precio $) /(1+$ inflación $)-1)$, se encuentra que el proyecto que tenía un VPN negativo de menos 2.902,81, tiene ahora un VPN positivo de 5.966,46. Este ejemplo está en el archivo mencionado, en la hoja PRECIOS RELATIVOS.

Se debe tener en cuenta que la relación entre la tasa de interés corriente y real o deflactada se establece por

$\left(1+\mathrm{i}_{\mathrm{c}}\right)=\left(1+\mathrm{i}_{\mathrm{r}}\right)\left(1+\mathrm{i}_{\mathrm{f}}\right)$

Donde:

$i_{c}=$ tasa de interés corriente

$i_{r}=$ tasa de interés real o deflactada

$i_{f}=$ inflación o componente inflacionaria

Entonces dos ingresos, uno a precios corrientes y otro a precios constantes o, lo que es lo mismo, sin aumento de precios, mantienen la siguiente relación si $\mathrm{i}_{\mathrm{f}}=\mathrm{i}_{\mathrm{a}} \mathrm{y}$ si todos los aumentos son iguales a la inflación:

$\mathrm{I}_{\mathrm{c}(\mathrm{t}+1)}=\mathrm{I}_{\mathrm{k}(\mathrm{t})}\left(1+\mathrm{i}_{\mathrm{a}}\right)$

$\mathrm{I}_{\mathrm{k}(\mathrm{t})}=\mathrm{I}_{\mathrm{c}(\mathrm{t}+1)} /\left(1+\mathrm{i}_{\mathrm{f}}\right)=\mathrm{I}_{\mathrm{k}(\mathrm{t})}\left(1+\mathrm{i}_{\mathrm{a}}\right) /\left(1+\mathrm{i}_{\mathrm{f}}\right)$

Al descontar estos flujos se tiene, $\mathrm{I}_{\mathrm{c}(\mathrm{t}+1)} /\left(\left(1+\mathrm{i}_{\mathrm{f}}\right)\left(1+\mathrm{i}_{\mathrm{r}}\right)\right)=\mathrm{I}_{\mathrm{k}(\mathrm{t})}\left(1+\mathrm{i}_{\mathrm{a}}\right)\left(\left(1+\mathrm{i}_{\mathrm{r}}\right)\left(1+\mathrm{i}_{\mathrm{f}}\right)\right)$

En este caso, la igualdad es válida si y sólo si $i_{\mathrm{a}}=\mathrm{i}_{\mathrm{f}}$ y $\mathrm{I}_{\mathrm{k}}(\mathrm{t})=\mathrm{I}_{\mathrm{k}}(\mathrm{t}+1)$ (esto significa precios constantes), o sea,

$\mathrm{I}_{\mathrm{c}(\mathrm{t}+1)} /\left(\left(1+\mathrm{i}_{\mathrm{f}}\right)\left(1+\mathrm{i}_{\mathrm{r}}\right)\right)=\mathrm{I}_{\mathrm{k}} /\left(1+\mathrm{i}_{\mathrm{r}}\right)$

Donde:

$I_{k}=\quad$ Ingreso neto a precios constantes

$I_{c}=$ Ingreso neto a precios corrientes o nominales

$i_{f}=$ Inflación

$i_{a}=$ Tasa de aumento en los precios

$i_{c}=$ Tasa de interés corriente o nominal
Esta es la razón por la cual algunos autores dicen que el VPN a precios constantes es idéntico al VPN a precios corrientes. Si las tasas de inflación y la de aumento de precios no son iguales, se deduce que habrá unas ganancias (en caso de que el aumento de precio de los ingresos sea mayor que la inflación o que el aumento de precio de los gastos sea menor que la inflación), o unas pérdidas (en caso de que el aumento de precio de los ingresos sea menor que la inflación o que el aumento de precio de los gastos sea mayor que la inflación), que no son tenidas en cuenta cuando se calcula el VPN. ${ }^{7}$

\subsubsection{Supuesto D}

Las ventas y los pagos por bienes y servicios se hacen de estricto contado

Esto, sobra decirlo, está lejos de ser cierto. Para resolver este punto se debe hacer el cambio propuesto arriba, de ajustar las cifras constantes (que se trasladen de un período a otro, esto es la cartera por cobrar), por la respectiva inflación. Esto, a su vez, implica que habrá que calcular y estimar la inflación futura, una de las ventajas del análisis a precios constantes.

Teniendo en cuenta las consideraciones anteriores se debe analizar qué sucede cuando se considera que los pagos o ingresos no son de contado. Es decir, cuando se reconoce que una empresa en su operación normal genera

Ganancias en el caso en que el aumento de precios de venta sea mayor que la inflación o que el aumento en precios de los insumos sea inferior a la inflación y pérdidas en el caso en que el aumento de los gastos sea mayor que la inflación, o que el aumento del ingreso sea menor que la inflación. 
cartera (cuentas por cobrar) y pasivos corrientes (cuentas por pagar). Entonces, si al pasar una suma de dinero de un período a otro (por ejemplo en el caso de la cartera pendiente o de los pagos a proveedores) no se ajusta por el factor de aumento o de inflación, el valor presente de esas sumas no será igual. Esto se ilustra mejor con el siguiente ejemplo:

\section{Ejemplo 3}

\section{Cuadro 10}

\begin{tabular}{|c|c|}
\hline Ventas 100\% de contado & Año 1 \\
\hline Precios corrientes \$ & 120 \\
\hline Precios constantes \$ & 100 \\
\hline Interés real & $12 \%$ \\
\hline Inflación o componente inflacionaria & $20 \%$ \\
\hline Tasa de descuento corriente & $1,12 \times 1,2-1=0,344$ ó 34,4\% \\
\hline Tasa de descuento constante & $12 \%$ \\
\hline Valor presente corriente \$ & $120 / 1.344=89.286$ \\
\hline Valor presente constante \$ & $100 / 1,12=89.286$ \\
\hline
\end{tabular}

Como se observa, el valor presente es igual ya que el aumento es igual a la inflación en este ejemplo $20 \%$. Si las ventas fueran a crédito inclusive en forma parcial, se tiene:

\section{Cuadro 11}

\begin{tabular}{|c|c|c|}
\hline Ventas 90\% de contado \$ & Año 1 & Año 2 \\
\hline Corrientes & 108 & 12 \\
\hline Constantes & 90 & 10 \\
\hline Valor presente corriente & $108 / 1.344=80.357$ & $12 /(1.344)^{2}=6.643$ \\
\hline Valor presente total & 87,0 & \\
\hline Valor presente constante & $90 / 1,12=80.357$ & $10 /(1,12)^{2}=7.972$ \\
\hline Valor presente total & 88.329 & \\
\hline
\end{tabular}

Como se observa, los dos valores no coinciden. Un análisis similar puede hacerse con el valor de salvamento. Por otro lado, si la relación de elasticidad se establece como la mayor o menor sensibilidad de la demanda a las variaciones de precio, al no existir un aumento de precios no se puede establecer una variación en la demanda ocasionada por el aumento de precios. La principal falla de este análisis a precios constantes es que los supuestos implícitos que hay detrás del modelo deforman la realidad que se desea representar; por tanto, el modelo no se puede validar al compararlo con la realidad.

Como se dijo arriba, si se corrige el movimiento del flujo de caja por la inflación, entonces sí coinciden los valores. Siguiendo con el mismo ejemplo, se tiene: 


\section{Cuadro 12}

\begin{tabular}{|c|c|c|}
\hline Años & $\mathbf{1}$ & $\mathbf{2}$ \\
\hline Corrientes & 108 & 12 \\
\hline Constantes & 90 & $10 /(1+20 \%)=8.333$ \\
\hline Valor presente corriente & 80.357 & 6.643 \\
\hline Valor presente total & 87.000 & \\
\hline Valor presente constante & 80.357 & 6.643 \\
\hline Valor presente total & 87.000 & \\
\hline
\end{tabular}

Aquí se consideró que el ajuste habría que hacerlo con la inflación. En estricto sentido, esos ajustes se deberían hacer con los cambios de precios previstos. En este ejemplo, se supone implícitamente que los cambios de precios son iguales a la inflación.

Para ratificar todo lo dicho se toma el ejemplo detallado y se calcula el VPN a precios constantes y se calcula el VPN con la tasa deflactada; luego, se incluyen los supuestos presentados arriba, tanto a precios constantes como a precios corrientes y se observa que esa es la única forma posible para que los resultados del VPN sean iguales. Y esto debe ser así, porque si no son iguales a precios corrientes y a precios constantes, basta que la inversión inicial varíe por el valor de la diferencia para que se cambie la decisión.

La importancia del hecho de no vender o pagar a crédito se puede apreciar con el análisis de sensibilidad presentado arriba. Para un aumento de $1 \%$ en la política de recaudos o de pagos (en lugar de $95 \%$ se utilizaría $95,95 \%$ ) el VPN aumentaría $0,64 \%$ y en el caso de los pagos se reduciría en $-1,17 \%$. Un análisis similar se haría con el valor de salvamento.

\subsubsection{Supuesto E}

No existe valor de salvamento del proyecto

Esto puede defenderse como una posición conservadora, pero muchos proyectos de inversión pueden depender del valor de salvamento al final del período de análisis. En caso de que se incluya, la relación entre en valor de salvamento a precios corrientes y a precios constantes debe ser idéntica a $\left(1+i_{f}\right)^{n}$, donde $i_{f}$ es exactamente la inflación; en general, esta proporción debe ser el aumento acumulado de la inflación, teniendo en cuenta que las tasas pueden ser diferentes.

\subsubsection{Supuesto F}

No existen relaciones de elasticidad precio-demanda

Si el analista trabaja con precios constantes y la elasticidad se define como la sensibilidad de la demanda a las variaciones de precio, entonces, como no hay aumento de precios, el modelo no captura estas variaciones, favorables o no. 
Para corregir esto, la función de elasticidad debe considerar inflación cero en el caso de precios constantes, y los aumentos de precios relativos deben ser iguales a:

$(1+$ aumento de precios a corrientes $) /\left(1+i_{f}\right)-1$

No deben calcularse, según costumbre arraigada, de la siguiente manera:

Aumento de precios a corrientes - inflación

De esta manera, el factor de elasticidad del ejemplo detallado se mantendría igual con ambas metodologías, asíi:

$$
\begin{aligned}
& 1-0,366\left[\frac{(1+\text { aumento de precios corrientes })}{\left(1+i_{f}\right)}-1\right] \text { para precios corrientes }= \\
& 1-0,366\left[\frac{\left(1+\left(1+i_{f}\right)(1+\text { aumento de precios relativos })-1\right)}{\left(1+i_{f}\right)}-1\right]= \\
& 1-0,366[(1+\text { aumento de precios relativos })-1]= \\
& 1-0,366 \text { ( aumento de precios relativos ) para precios constantes }
\end{aligned}
$$

\subsubsection{Supuesto $G$}

La tasa de descuento a precios corrientes o nominales debe ser exactamente igual a $\left(1+i_{p}\right)(1+i)-1$ y a precios constantes debe ser igual a $i_{r}$, la tasa de interés real

Esta suposición se puede compensar teniendo en cuenta que se debe deflactar -con la tasa de inflación- las tasas de descuento corrientes, que incluyen el riesgo que otros -los acreedores y los accionistas- perciben en la firma. Por tanto, la tasa de descuento a precios constantes no sería $i_{r}$, sino,

$\left(1+i_{c}\right) /\left(1+i_{f}\right)-1$

En rigor, hay que hacer los ajustes correspondientes al endeudamiento, así:

$\left(1+i_{c}\right) /\left(1+i_{f}((1-T) * D / A+(1-D / A))\right)-1$

Donde

$\mathrm{i}_{\mathrm{c}}=$ tasa de descuento a precios corrientes

$\mathrm{i}_{\mathrm{f}}=$ tasa de inflación
$\mathrm{T}=$ tasa de impuestos

$\mathrm{D}=$ pasivos

$A=$ total de activos

En esta expresión lo que se tiene en cuenta es el efecto de la proporción deuda patrimonio y los impuestos.

\subsubsection{Supuesto $H$}

El saldo del período del FT (ingresos menos egresos) después de inversión de excedentes debe considerase parte del FCF como si fuera una recompra de acciones o participaciones de los socios

Esto debe ocurrir para evitar que exista un excedente depositado en caja. Si se deposi-

Esta es la función de elasticidad hipotética, utilizada en el ejemplo detallado. 
ta en caja no gana intereses y eso desvirtúa el VPN calculado. En nuestro ejemplo este supuesto no afecta el resultado porque el valor de ese saldo es cero.

Estas ocho condiciones o supuestos se deben cumplir para que los valores del VPN calculado con ambas metodologías coincidan. Como se puede intuir, los ajustes que se necesitan para que coincida el análisis a precios constantes con el análisis a precios corrientes son tales que anulan la supuesta simplicidad que pretende tener el método; entre otras cosas, porque termina siendo necesario proyectar tanto la inflación como los aumentos de precios. La conclusión es que se debe trabajar a precios corrientes y no a precios constantes.

\section{Evaluación a precios corrientes y constantes}

En este aparte se ilustra la evaluación del proyecto a precios corrientes y constantes y se establecen las diferencias en los resultados.

\section{Ejemplo 4}

En el archivo INFLACIÓN.XLS hay un ejemplo. Allí se prevén unas tasas de aumento de precios para diferentes variables y las tasas de aumento de precios se fijan en cero, esto es, se evalúa el proyecto a precios constantes.

A precios corrientes el FLUJO DE CAJA LIBRE resultante es:

\section{Cuadro 13}

\begin{tabular}{|c|r|r|r|r|c|}
\hline Años & $\mathbf{0}$ & $\mathbf{1}$ & $\mathbf{2}$ & $\mathbf{3}$ & $\mathbf{4}$ \\
\hline $\begin{array}{c}\text { Flujo de caja del proyecto } \\
\text { después de impuestos }\end{array}$ & $-12.000,00$ & 0,00 & 506,83 & $1.057,32$ & $25.689,72$ \\
\hline Tasas de descuento & & $34,01 \%$ & $34,02 \%$ & $31,40 \%$ & $30,10 \%$ \\
\hline Factor de valor presente & & 0,7462 & 0,5568 & 0,4237 & 0,3257 \\
\hline $\begin{array}{c}\text { VPN (tasa(s) de descuento) } \\
\text { después de impuesto }\end{array}$ & $-2.902,81$ & & & & \\
\hline
\end{tabular}

Ahora se debe observar la diferencia de resultados al evaluar el proyecto a precios constantes. Cuando los aumentos de precios son $0 \%$ y no se hacen los demás ajus- tes a los supuestos implícitos, o sea que se trabaja a precios constantes, vale decir, aumento de precios $0 \%$ y tasa de descuento real o deflactada, el FCL es:

\section{Cuadro 14}

\begin{tabular}{|c|c|c|c|c|c|}
\hline Años & $\mathbf{0}$ & $\mathbf{1}$ & $\mathbf{2}$ & $\mathbf{3}$ & $\mathbf{4}$ \\
\hline FCL después de impuestos \$ & $-12.000,00$ & 0,00 & 292.09 & 499.96 & $19,235.04$ \\
\hline Tasas de descuento & $6 \%$ & $6 \%$ & $6 \%$ & $6 \%$ & $6 \%$ \\
\hline Factor de valor presente & 1,0000 & 0,9434 & 0,8900 & 0,8396 & 0,7921 \\
\hline VPN (tasa(s) de descuento) $\$$ & $3.915,69$ & & & & \\
\hline
\end{tabular}


En la evaluación a precios corrientes el VPN fue negativo y se rechazó, este valor contradice la decisión a precios constantes. O sea, que un proyecto malo en la realidad (precios corrientes) puede ser aceptado como bueno a precios constantes, porque el VPN es positivo.

El mayor peso en esta distorsión lo tiene el efecto de la elasticidad (al tener $0 \%$ de aumento, el modelo hace crecer la demanda).
Sin embargo, cuando se anula el efecto de la elasticidad, el VPN del análisis a precios constantes también es positivo con valor de $4.675,67$.

Al incluir los supuestos presentados arriba (véase hojas FLUJO DE CAJA CTE y FLUJO DE CAJA K del archivo INFLACION.XLS) se obtienen los siguientes flujos de caja a precios corrientes y constantes.

\section{Ejemplo 5}

\section{Cuadro 15}

\begin{tabular}{|c|c|c|c|c|c|}
\hline \multicolumn{7}{|c|}{ Precios corrientes con todos los supuestos } \\
\hline Años & $\mathbf{0}$ & $\mathbf{1}$ & $\mathbf{2}$ & $\mathbf{3}$ & $\mathbf{4}$ \\
\hline FCL después de impuestos \$ & $-12.000,00$ & $1.305,68$ & $4.850,06$ & $6.588,47$ & 0,00 \\
\hline Factor de valor presente & 1,0000 & 0,7733 & 0,5931 & 0,4624 & 0,3635 \\
\hline $\begin{array}{c}\text { VPN (tasa(s) de descuento) } \\
\text { antes de impuesto \$ }\end{array}$ & $-5.067,20$ & & & & \\
\hline
\end{tabular}

\begin{tabular}{|c|c|c|c|c|c|}
\hline \multicolumn{7}{|c|}{ Precios constantes con todos los supuestos } \\
\hline & Año 0 & Año 1 & Año 2 & Año 3 & Año 4 \\
\hline FCL después de impuestos \$ & $-12.000,00$ & $1.070,23$ & $3.232,08$ & $3.628,56$ & 0,00 \\
\hline Factor de valor presente & 1,0000 & 0,9434 & 0,8900 & 0,8396 & 0,7921 \\
\hline $\begin{array}{c}\text { VPN (tasa(s) de descuento) } \\
\text { antes de impuesto \$ }\end{array}$ & $-5.067,20$ & & & & \\
\hline
\end{tabular}

Como se observa, en este caso los VPN sí son idénticos, y deben serlo. En el archivo INFLACION.XLS se encuentra el ejemplo detallado a precios constantes y tasa de descuento sin inflación y el mismo ejemplo detallado pero con los supuestos planteados arriba, tanto con precios corrientes como constantes, de donde se extrajeron los flujos de caja presentados en este ejemplo.

\section{Evaluación con precios relativos}

Si se toman los aumentos de precios relativos y se descuenta a la tasa de interés real, entonces se deben hacer los siguientes ajustes:

i) El aumento de precios relativo es (1+aumento de precio)/(1+tasa de inflación) -1 . En este ejemplo serían: 


\section{Cuadro 16}

\begin{tabular}{|c|c|c|c|}
\hline 11 Aumentos en precios de compra & $0.82 \%$ & $0.81 \%$ & $0.83 \%$ \\
\hline 13 Aumentos en precios de venta & $3.28 \%$ & $1.63 \%$ & $1.65 \%$ \\
\hline 16 Aumentos en honorarios & $2.46 \%$ & $0.00 \%$ & $0.00 \%$ \\
\hline 18 Aumentos en gastos generales & $0.82 \%$ & $0.81 \%$ & $1.65 \%$ \\
\hline 20 Aumentos en mano de obra & $4.10 \%$ & $0.81 \%$ & $0.83 \%$ \\
\hline
\end{tabular}

ii) La tasa de descuento a precios relativos tiene que ser exactamente igual a $\mathrm{i}_{\mathrm{r}}$, la tasa real de interés. El factor de valor presente se calcula con $6 \%$ como tasa de descuento. iii) La función de elasticidad debe cambiarse a:

1-0,366 x aumento relativo de los precios

Con todos estos ajustes, entonces el flujo de caja libre y el VPN son:

\section{Cuadro 17}

\begin{tabular}{|c|c|c|c|c|c|}
\hline Años & $\mathbf{0}$ & $\mathbf{1}$ & $\mathbf{2}$ & $\mathbf{3}$ & $\mathbf{4}$ \\
\hline FCL & $-12.000,00$ & 0,00 & 396,27 & 687,74 & $21.225,74$ \\
\hline Factor de valor presente & 1,0000 & 0,9434 & 0,8900 & 0,8396 & 0,7921 \\
\hline VPN & $5.742,89$ & & & & \\
\hline
\end{tabular}

El VPN es ahora positivo y un proyecto malo se aceptaría como bueno. Una situación similar a la que se encontró a precios constantes.

\section{Evaluación en moneda extranjera}

Algunos proponen trabajar en dólares y sobre la base de que la inflación en Estados Unidos es casi cero, se piensa que estos problemas se eliminan. Esto es cierto sólo en el caso en que todos los ingresos y egresos ocurran en dólares o que los aumentos de precios de los rubros que se manejan en pesos coincidan con la devaluación. Es fácil demostrar que si, por ejemplo, la nómina que se maneja en pesos se aumenta en $25 \%$ y la devaluación es de $15 \%$, entonces al considerar los rubros en dólares constantes se estaría despreciando un sobrecosto, así: 


\section{Ejemplo 6}

\section{Cuadro 18}

\begin{tabular}{|c|c|c|c|}
\hline Años & $\mathbf{1}$ & $\mathbf{2}$ & $\mathbf{3}$ \\
\hline Nómina \$ & 10.000 .000 & 12.500 .000 & 15.000 .000 \\
\hline $\begin{array}{c}\text { Tasa de cambio \$/US\$ } \\
\begin{array}{c}\text { Nómina a precios constantes en } \\
\text { dólares US\$ }\end{array}\end{array}$ & 1.000 & 1.150 & 1.300 \\
\hline $\begin{array}{c}\text { Nómina a precios de cada año en } \\
\text { dólares US\$ }\end{array}$ & 10.000 & 10.000 & 10.000 \\
\hline Aumento de la nómina en dólares & & $10.869,57$ & $11.538,46$ \\
\hline
\end{tabular}

Este sencillo ejemplo muestra cómo al considerar dólares constantes, cuando una partida se maneja en pesos, puede también conducir a distorsiones graves.

\section{Otras consideraciones}

Además de los problemas arriba mencionados, se deben tener en cuenta los siguientes aspectos:

i) La realidad se refiere a que los datos de los cuales dispone el analista son los precios y costos históricos (corrientes) y las tasas de interés históricas (corrientes); por otro lado, dispone también de las series históricas de los diferentes indicadores económicos que le permitirán hacer las proyecciones necesarias. Y al mencionar proyecciones no se alude sólo a proyecciones estadísticas, sino también cálculos calificados, basados en el pasado, el presente y en cualquier información disponible al analista.

ii) En muchos cursos de posgrado y de ejecutivos se oye decir que al descontar los flujos de caja a precios corrientes se está inflando el VPN. Esta aprecia- ción es incorrecta. Cuando los flujos de caja se descuentan a la tasa de descuento corriente, el efecto de la inflación se elimina porque la tasa de descuento tiene incluida una componente inflacionaria. Entonces, cuando se aplica el proceso de descuento a las cifras en precios corrientes, el efecto de la inflación se elimina. Y el VPN resultante está deflactado en términos de inflación.

iii) Un proyecto financiado debe asumir costos financieros, en lo posible inmodificables, cualquiera que sea el nivel de inflación; entonces, al considerar flujos de caja a precios constantes se estaría favoreciendo el proyecto al imputársele de manera explícita a través de los desembolsos de la financiación su efecto en los impuestos, el cual resulta en unos ahorros en impuestos relativamente mayores que a precios corrientes. Este análisis es similar al presentado arriba sobre la depreciación. Dichos pagos de intereses, al igual que la depreciación, no se modifican al definir precios o pesos constantes.

iv) Existe un tabú en cuanto al pronóstico de la inflación se refiere; sin embargo, 
se realizan predicciones implícitas o explícitas acerca de niveles de ventas, fenómeno afectado por variables tan impredecibles como los niveles de la economía y de precios, acciones de la competencia, fenómenos atmosféricos, políticas gubernamentales, etcétera.

v) Así mismo, se tiene la ilusión de que un pronóstico de ciertas variables (nivel de ventas o de producción, por ejemplo) es confiable; en rigor se deben utilizar varios pronósticos y combinar los posibles resultados, y para esto se pueden utilizar métodos de simulación. Si se considera que la variación en los precios, o inclusive, la inflación, son fenómenos probabilísticos, se pueden establecer probabilidades históricas o subjetivas para diferentes niveles de precios y a partir de allí hacer cálculos probabilísticos sobre los precios que ocurrirán en el futuro; si se considera que los precios obedecen a políticas gubernamentales, variables macroeconómicas, entre otras, se pueden hacer predicciones de la misma forma como se predice el comportamiento del consumidor, dadas ciertas políticas oficiales supuestas, o sea lo que se conoce como análisis de escenarios, para lo cual existen expertos sobre el tema. Se debe reconocer que pronosticar precios es una tarea difícil, ya que puede depender en parte de las medidas de política económica del gobierno de turno y en ocasiones de factores netamente políticos y sociales entre otros. En otras palabras, los precios involucrados en el proyecto que se analiza, son otras variables que hay que calcular y su resultado adolecerá de las fallas de cualquier pronós- tico, puesto que ninguno es exacto. $\mathrm{La}$ toma de decisiones es un proceso con resultados difícilmente reversibles, basado en datos incompletos e inexactos; a pesar de ello, es necesario tomar decisiones y ponerlas en marcha. A veces es preferible hacer proyecciones basadas en juicios basados en la experiencia, que hacer pronósticos fundamentados en modelos estadísticos. Esto es cierto, sobre todo cuando la economía cruza coyunturas muy críticas.

vi) Aceptado el hecho de que el aumento de precios es difícil de pronosticar, se puede considerar la alternativa, con el grado de complejidad que se desee, de incluirlo en un modelo de evaluación de inversiones basado en métodos de simulación o en el análisis de sensibilidad. En el peor de los casos, se puede optar por la versión más primitiva del análisis de sensibilidad en la cual se consideran diferentes niveles de los valores de las variables, que es posible con cualquier hoja de cálculo.

vii) Un proyecto debe ser controlado en su ejecución, por tanto los valores que se van a controlar deben ser similares a los que ocurran en la realidad. En otras palabras unos estados financieros a precios o pesos constantes de nada sirven para fines de control pues los valores a controlar nunca serán ni siquiera parecidos a los pronosticados.

viii) Un PyG a precios constantes es una colcha de retazos donde se encuentran partidas deflactadas o a precios constantes y partidas no deflactadas, por 
ejemplo, los intereses, como ya se estudió o la depreciación, que no tiene sentido deflactarla.

ix) Hay que reconocer que los métodos de pronósticos, por lo general, implican que el contexto de donde salieron los datos históricos ocurrirá otra vez en el futuro. Es preferible utilizar varios pronósticos y la experiencia, la intuición y el conocimiento del entorno económico que tenga el analista o el decisorio, para hacer cálculos aproximados, pero que tienen un sustento subjetivo. Hemos estado anclados a procedimientos que eran válidos hace un siglo o menos, cuando los recursos de cálculo eran precarios. Hoy en día se cuenta con hojas de cálculo electrónicas y otro tipo de programas que permiten hacer análisis de escenarios, simulaciones de Monte Carlo, etcétera. Por tanto, se debe eliminar el temor a hacer pronósticos, incluyendo la inflación. La idea no es minimizar lo difícil que implica el trabajar con el futuro. Eso no es fácil. Pero hoy se cuenta con muchas más herramientas que antes para analizar los pronósticos. En otras palabras, los precios son algunas de las variables que hay que pronosticar. Hay que aceptar que cualquier método de pronóstico conlleva un cierto margen de error y hay que aprender a vivir con él.

x) Si ninguno de los anteriores argumentos fuera aceptable no se debe olvidar que para cualquier proyecto es necesario predecir las necesidades de fondos futuros para negociarlos con el banco con suficiente anticipación. Así mismo, para hacer cálculos de las necesidades de fondos es necesario proyectar las cifras a precios corrientes; de otra manera se estipularían necesidades de financiación a pesos constantes, lo cual no tiene ninguna ventaja práctica, ni ningún sentido en términos de la administración financiera. En otras palabras, el proyecto no debe evaluarse sólo desde el punto de vista de su conveniencia económica y financiera; se deben considerar también otros aspectos, como la viabilidad del proyecto en términos de liquidez. $\mathrm{O}$ sea, un proyecto puede ser recomendable desde el punto de vista económico y no ser factible por no contar con los recursos necesarios para llevarlo a cabo; la evaluación a precios constantes no tendría en cuenta esta situación ya que no tiene sentido establecer necesidades de recursos a precios constantes. Al comparar necesidades de liquidez para pagar préstamos contratados, inclusive en el instante cero, no se podría saber si el proyecto puede o no pagarlo, porque la liquidez a precios constantes no dice nada acerca de la disponibilidad real de dinero. Esto, aparte de la dificultad de definir bien las necesidades de fondos. No se debe olvidar que el flujo de tesorería queda distorsionado con los impuestos subestimados a precios constantes. Un flujo de tesorería proyectado a precios constantes no significa nada para la gerencia. Estos flujos de caja quedan distorsionados por muchos motivos, pero el más importante es la subvaloración de los impuestos, como ya se demostró. 


\section{Propuesta}

Lo que se propone es realizar la evaluación de alternativas de inversión a precios corrientes y no a precios constantes.

Para efectos de claridad es importante indicar los diferentes datos que se poseen y que deben proyectarse al hacer el análisis de una inversión a precios corrientes.
Se tienen:

- Series históricas de precios corrientes para cada clase de ingresos y de costos

- Unidades proyectadas

- Series históricas de intereses corrientes

- Se proyectan las diferentes series, ya sea en forma matemática o subjetiva, y se calcula el valor presente neto a la tasa de interés de descuento corriente

Al aplicar esas ideas, el valor presente neto del proyecto aparecerá así:

$$
V P N=\sum_{t=0}^{\infty} \frac{\left[\sum_{j=1}^{m} I_{j t}\left(1+h_{j}\right)^{t}-\sum_{k=1}^{n} E_{k t}\left(1+h_{k}\right)^{t}\right]}{\left(1+i_{c}\right)^{t}}
$$

Donde:

$m=$ Número de clases de ingresos

$n=\quad$ Número de clases de egresos

$E_{\mathrm{kt}}=$ Egresos de la clase $\mathrm{k}$ en el período t, precios corrientes

$I_{j t}=$ Ingresos de la clase $\mathrm{j}$ en el período $\mathrm{t}$, precios corrientes

$h_{\mathrm{j}}=$ Índice para calcular los ingresos clase $\mathrm{j}$, constante

$h_{\mathrm{k}}=$ Índice para calcular los egresos clase $\mathrm{k}$, constante

$i_{c}=$ Tasa de interés corriente, constante

La suposición de mantener $h_{j} y h_{k}$ constantes a lo largo del horizonte de planteamiento se puede eliminar con facilidad escribiendo:

$$
\prod_{i=0}^{t}\left(1+h_{i j}\right) \text { en lugar de } \sum\left(1+h_{j}\right)^{t} \text { etcétera }
$$

Al hacer el análisis en pesos corrientes, se trata de modelar o predecir la realidad futura y al efectuarlo en pesos constantes, se tienen unos supuestos completamente alejados de la realidad, como se expresó antes. Se puede decir que la evaluación a precios constantes es ciega ante los efectos que sobre la bondad o inconveniencia de un proyecto pueden tener los cambios de precios previstos. 


\section{Conclusiones}

Se ha demostrado que no es correcto trabajar con pesos constantes al hacer el análisis económico de alternativas, puesto que el modelo desvirtúa la realidad que pretende ilustrar, y los resultados son diferentes en la forma como en la práctica se trabaja en la evaluación de proyectos de inversión. En Vélez (1981) también se ilustra con un ejemplo que no es equivalente trabajar en pesos constantes y pesos corrientes.

La mayor falla de la metodología de precios constantes es que las suposiciones o condiciones implícitas que tiene distorsionan la realidad que se desea representar por medio del modelo (el flujo de caja y los estados financieros son un modelo). Por tanto, la validación de ese modelo con la realidad es imposible. La situación es, pues, muy simple: no es cierto que evaluar proyectos con precios y pesos constantes sea equivalente a evaluarlo con precios nominales o corrientes. Las metodologías de precios y pesos constantes son sesgadas y sobrevalúan un proyecto. Estas metodologías simplifican en exceso la realidad y producen resultados indeseables. $\mathrm{La}$ metodología correcta es la de precios corrientes; cualquier otro enfoque que no represente la realidad lo más cercano posible, se debe desechar en forma inmediata.

\section{Referencias bibliográficas}

Brealey, Richard A.; Stewart, C.; Myers; Alan, J.; Marcus, 1995. Fundamentals of corporate finance, McGraw-Hill, Publicado en español como Fundamentos de finanzas corporativas, McGraw-Hill.
Canada, J. R.; White, Jr., J.A., 1980. Capital Investment Decision Analysis for Management and Engineering, Prentice Hall, Englewood Cliffs, N. J.

Copeland, Thomas E.; Koller, T.; Murrin, J. 1995. Valuation: Measuring and Managing the Value of Companies, 2 ed., John Wiley and Sons.

Coss, Bu R. 1981. Análisis y evaluación de proyectos de inversión, Limusa, México.

Damodaran, Aswath, 1996. Investment Valuation, John Wiley.

Dixon, John A.; Hufschmidt, Maynard M. Eds., 1986. Economic Valuation Techniques for the Environment. A Case Study Workbook, The John Hopkins University Press.

Levy, Haim; Marshall Sarnat, 1982. Capital Investment and Financial Decisions, 2 ed., Prentice Hall.

Lora, Eduardo, 1987. Técnicas de medición económica. Metodología y aplicaciones en Colombia. TM Editores - Fedesarrollo, Bogotá.

Puig-Andreu, J.V.; Renau-Piqueras, J.J., 1981. Análisis y evaluación de proyectos de inversión, Editorial Hispano Europea, S. A., Barcelona.

Van Horne, J.C., 1998. Financial Management and Policy, 11 ed., Prentice Hall, Englewood Cliffs, New Jersey.

Vélez Pareja, Ignacio, 2001. Decisiones de inversión. Enfocado a la valoración de empresas $2^{a}$ ed., Ceja, (disponible en: http://www.javeriana. edu.co/decisiones/libro on_line). 
Vélez Pareja, Ignacio, 1994, Evaluación financiera de proyectos de inversión, Superintendencia del Subsidio Familiar, Bogotá.

, 1981. Modelos de reemplazo: Un ejemplo de tecnología inapropiada, Universitas Económica, VIII, n. 1. diciembre, p. 103-131, (También en: Vélez, Ignacio, 1983. Nota: Replacement models: Technology innapropriate to nonindustrialized countries, Interfaces, v. 13, n. 5, octubre, p. 122- 123).
Vélez Pareja, Ignacio, 1999. "Project Evaluation in an Inflationary Environment", Social Science Research Network, Finance Teaching and Case(case) v. 4, n. 2, en Corporate Finance Abstracts Valuation, Capital Budgeting and Investment Policy (wps) v. 2, n. 6, http: // papers.ssrn.com/paper.taf?abstract_id=148410.

Weston, J.; Fred; Copeland, T. E., 1992, Managerial Finance, 9 ed. The Dryden Press, Hay traducción en español como Finanzas en Administración, 9 ed., McGraw-Hill. 